\title{
Adjuvant therapies for large bowel cancer
}

\author{
Wasantha Rathnayake, MD \\ Consultant Clinical Oncologist, National Cancer Institute, Maharagama, Sri Lanka.
}

Key words: Large bowel; Cancer; Adjuvant chemotherapy.

Large bowel cancer is one of the most common malignancies, especially in Western countries, accounting for over a million new cases and about 500,000 deaths per year, worldwide. In Sri Lanka even it was the third most common malignancy among male and fifth among female in 2009. Surgery is the primary curative modality with the intent of 80 percent in patients with localized colorectal adenocarcinoma. However, the risk of recurrence is still high as 50 percent patients after potentially curative surgery. Adjuvant chemotherapy for patients with localized colon cancer is used in order to eradicate micro metastases and, therefore, to improve the survival rate after curative surgical resection. Chemotherapy is the principal adjuvant therapy for patients with colon cancer and the addition of radiotherapy has not been shown to improve survival. Benefits of adjuvant chemotherapy for rectal cancer are extrapolated from colonic cancer as there is no direct evidence to support its use in postoperative adjuvant chemotherapy regimes. Recent randomized trials, incorporated new drugs, such as capecitabine, irinotecan and oxaliplatin into the adjuvant setting, leading to significant alterations in patient care.

There are many controversies in adjuvant therapy. In this review, I have considered these areas such as Chemotherapy in stage III? Chemotherapy in stage II? How should we give 5FU? What's better than 5FU? What about Novel Agents? Can Capecitabine substitute for $5 F U$ ? Should we treat rectal cancer like colon cancer? What is optimal duration of chemotherapy?

\section{Adjuvant therapy in stage iii colon cancer}

The issue of adjuvant chemotherapy after surgery in colon cancer remained controversial until the late 1980s. In 1988 a meta-analysis, which included nearly 10,000 patients in 25 randomized trials revealed a small survival benefit in patients receiving adjuvant 5-FU after surgery compared to those who underwent surgery alone [1].

Furthermore, Levamisole (LEV), an immunomodulator

Correspondence: Wasantha Rathnayake, MD

Consultant Clinical Oncologist, National Cancer Institute, Maharagama.

E-mail: wasantha71@yahoo.com with mild toxicity, showed a small but statistically significant survival benefit as a single agent in adjuvant therapy. The impact of the combination of 5-FU and LEV was tested through a large randomized trial, the Intergroup 0035 (Int 0035) trial with stage III colon cancer, to observation or to treatment for one year with 5-FU/LEV combination. Patients with stage $\mathrm{C}$ disease also randomly assigned for treatment with LEV alone (Fig-1).

$$
5 F U+\text { Lev vs. Surgery Only }
$$

Stage III Resected
Colon Cancer
No Treatment
Levamisole

Figure 1. Trial of levamisole with 5FU for stage 111 Colon Cancer

Among patients with stage $\mathrm{C}$ disease, the risk of cancer recurrence was reduced by $41 \%$ for the LEV plus 5 -FU arm, compared with the observation only arm [2]. The overall death rate was reduced by $33 \%$. Treatment with LEV alone had no detectable effect. Following this trial Fluorouracil and Levamisole were recommended as standard therapy in resected stage III colon cancer.

Leucovorin(LV-Folinic acid) is a reduced folate that increases the antitumor activity of 5-FU through enhancement of the inhibition of thymidylate synthase. To evaluate the efficacy of the combination of 5-FU and LV as adjuvant therapy, The International Multicentre Pooled Analysis of Colon Cancer Trials (IMPACT) pooled data for combined analysis from three separate trials of 5-FU and LV versus surgery only in stage II and III colon cancer patients [3]. The 5-FU/LV combination significantly reduced mortality by $22 \%$ and relapses by $35 \%$, increasing 3 -year relapse-free survival from $62 \%$ to $71 \%$ and overall survival from $78 \%$ to $83 \%$.

The next step was to determine if 5-FU/LV was superior to 
5-FU/LEV in the adjuvant setting. In addition, the optimal duration of treatment was in question. An Intergroup study (INT-0089) randomized 3,759 patients with stage III and high-risk stage II colon cancer to

5-FU/LEV for 12 months, weekly 5-FU with high-dose LV (5-FU 500 mg/m2, LV 500 mg/m2, Roswell-Park regimen) for 7 to 8 months, 5-FU with low-dose LV (5-FU $425 \mathrm{mg} / \mathrm{m} 2$, LV $20 \mathrm{mg} / \mathrm{m} 2$, known as the Mayo Clinic regimen) for 6 months, or 5-FU/LEV for 6 months (4). Results from this trial showed that the: (i) 5-FU/low dose LEV is equivalent to the 5-FU/high dose LV; (ii) 5-FU/LV given for 6 months is as good as given for 12 months; (iii) there is no significant difference between the two most commonly used bolus 5-FU/LV dose schedules: This resulted in the acceptance of 5-FU/LV for 6 to 8 months as the standard treatment for stage III colon cancer since middle of 90's.

The next step in the evolution of adjuvant chemotherapy for colon cancer was the evaluation of the 5-FU/LV continuous infusion. Continuous infusion of 5-FU resulted in less haematological toxicities and a small, but statistically significant survival advantage over bolus regimens in advanced CRC, thus, providing the rationale to investigate continuous infusion 5-FU as adjuvant therapy [5].

Continuous infusion of 5-FU has been compared to bolus administration as adjuvant treatment in patients with colon cancer in three randomized phase III trials (SAFFA Trial, DeGramont vs. Mayo,Intergroup 0153) (fig-2a and b). None of these studies yielded a significant difference in terms of DFS and OS in favour of any arm. However, continuous infusion 5-FU had a more favourable profile concerning toxicity. This resulted in the acceptance of Infused

\section{DeGramont vs. Mayo}

Stage II / III Colorectal Cancer

\begin{tabular}{|c|c|}
\hline $\begin{array}{c}\text { DeGramont } \\
24 \text { weeks }\end{array}$ & $\begin{array}{c}\text { Mayo } \\
24 \text { weeks }\end{array}$ \\
\hline DeGramont \\
36 weeks \\
\hline
\end{tabular}

Figure 2a. Comparison of De Gramont vs. Mayo Trial

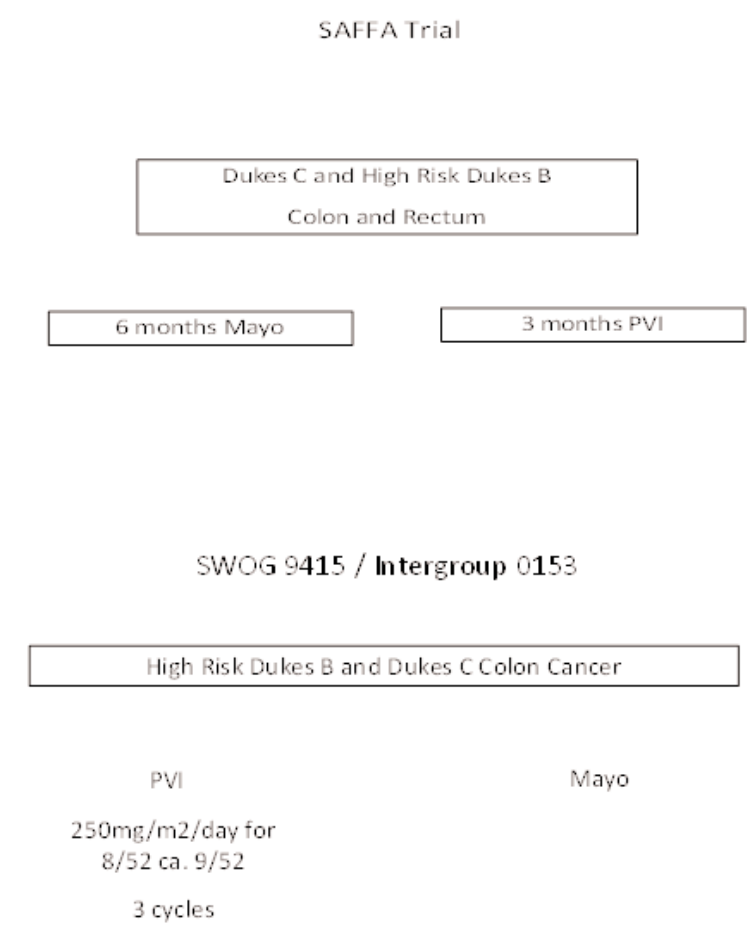

5FU as effective as bolus 5FU

Figure 2b. Comparison of De Gramont vs. Mayo Trial

How long should we give 5FU for? A study by Saini et al [6] has demonstrated that the administration of infused 5FU as adjuvant therapy is associated with less acute toxicity and less impairment of quality of life than a standard bolus schedule. Furthermore, this has been achieved without any obvious adverse effect on outcome, and indeed there appears to be improved outcome, in the group of patients with rectal cancer. Reducing treatment duration to 12 weeks rather than six months was not associated with any detrimental effects on the efficacy of infused 5-FU.

Capecitabine (Xeloda-oral fluropiramidine) is a prodrug that undergoes a three-step enzymatic conversion to fluorouracil. Capecitabine was studied as an adjuvant treatment for patients with resected Dukes' C colon cancer in the X-ACT trial [7]. Patients were randomly assigned to receive the Mayo Clinic regimen or capecitabine 2500 $\mathrm{mg} / \mathrm{m} 2$, for two consecutive weeks, followed by one week rest. After a median follow-up period of 3.8 years, capecitabine showed a trend towards superior DFS versus 5-FU/LV, and a similar trend to superiority for OS. Relapse-free survival (RFS) was also superior for capecitabine compared to 5-FU/LV. Consequently, Capecitabine considered as effective as 5FU in the adjuvant therapy of stage III colon cancer patients. 
What is better than 5FU?, The European Multicenter International study of oxaliplatin/infusional 5-FU/LV [FOLFOX 4] in the adjuvant treatment of Colon Cancer (MOSAIC) study(fig-3) randomized 2,246 stage II and III colon cancer patients to receive 5-FU/LV (LV $200 \mathrm{mg} / \mathrm{m} 2$ followed by 5 -FU bolus $400 \mathrm{mg} / \mathrm{m} 2$ and then a 22-hour infusion of 5- FU $600 \mathrm{mg} / \mathrm{m} 2$ given on 2 consecutive days every 14 days, for 12 cycles), or the same 5-FU/LV regimen plus oxaliplatin, (85 mg/m2). The 3-year DFS was $78.2 \%$ in the oxaliplatin/5-

FU/LV group and $72.9 \%$ in the 5 -FU/LV group $(\mathrm{p}=0.002)$ for stage III disease but there was no conclusive evidence for stage II disease, while the 3-year OS was $87.7 \%$ and $86.6 \%$, respectively [8].

Resected Stage II / III Colon Cancer

\section{5-FU/LV \\ Oxaliplatin $85 \mathrm{mg} / \mathrm{m} 2$}

Figure 3. Detail of the MOSAIC Trial

Furthermore, one recently reported study (NSABP C-07) has also shown that the addition of oxaliplatin to bolus 5FU/LV significantly improved DFS compared to bolus 5FU/LV alone [9]

It was concluded, oxaliplatin-containing regimen should be considered for all stage III colon cancer patients. However, there is no overall survival advantage has been yet demonstrated in either MOSAIC or NSABP C-07 studies, as data are still maturing.

In contrast with the oxaliplatin studies, three randomized trials comparing irinotecan/5-FU combinations with bolus or continuous infusion 5-FU/LV failed to show any benefit in terms of DFS in favor of irinotecan $[10,11]$.

$\begin{array}{ll}\text { CALGB 89803 } & \text { ASCO } 2004 \\ \text { ACCORD } 2 & \text { ASCO 2005 } \\ \text { PETACC } 3 & \text { ASCO 2005 }\end{array}$

Therefore it was concluded that adjuvant studies with irinotecan based regimens show no benefit or not adequately designed hence value of adjuant irinotecan is still to be proven. Next question was, can capecitabine substitute for 5FU? XELOX-A a Phase III trial of capecitabine plus oxaliplatin showed improved disease free survival as adjuvant therapy for stage III colon cancer [12]. So it was concluded capecitabine can replace 5FU in combination regimen too.

\section{Adjuvant therapy in stage ii colon cancer}

The role of adjuvant chemotherapy in stage II colon cancer remains a controversial issue.

The relative and absolute benefits of adjuvant chemotherapy in stage II colon cancer have been suggested from a combined analysis from NSABP C01-C04 and QUASAR trials that compared different adjuvant chemotherapy regimens with each other or with no adjuvant treatment.

The QUASAR study randomized a total of 3,239 patients (91\% stage IIB) to treatment with 5-FU/LV, with or without levamisole, (Mayo or Roswell Park, 6 months; $\mathrm{n}=$ $1,622)$ or observation only $(n=1,617)$ after curative resection [13]. Treatment of patients with 5-FU/lev significantly increased 5-year survival by approximately 3 percentage points as compared with that in patients observed after surgery ( $80.3 \%$ vs. $77.4 \%)$.The findings of QUASAR marked the first demonstration of a statistically significant survival benefit for adjuvant chemotherapy in stage II patients (fig-4).

Figure 4. Details of the QUASAR Randomised trial

\section{QUASAR}

Completely resected CRC

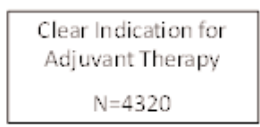

$5 F U 370 \mathrm{mg} / \mathrm{m} 2+L D / H D F A$ 6xMAYO vs 30 weekly
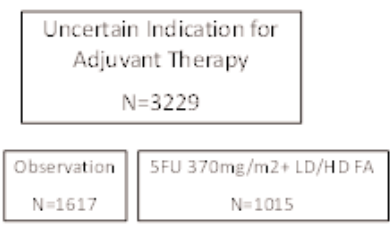

The MOSAIC trial had stage II tumors $(\mathrm{n}=899)$ and were randomized to receive FOLFOX4 $(\mathrm{n}=451)$ or LV5FU2 (n $=448$ ) treatment [8]. A total of $87 \%$ of patients with stage II disease treated with FOLFOX4 were disease-free 3 years post therapy compared with $84.3 \%$ of those treated with LV5FU2.This represented an improvement of approximately $3 \%$ in DFS and a $20 \%$ recurrence risk reduction in the FOLFOX4 treatment arm.

Subgroup analysis of patients with clinical markers of poor prognosis (T4 stage, bowel obstruction, tumor perforation, poorly differentiated tumor, lymphovascular invasion, fewer than 10 sampled lymph nodes) found that high-risk stage II patients treated with oxaliplatin experienced an improvement in DFS of 5.4\%. 
IMPACT B2: 5FU+FA in Dukes' B

1016 eligible patients with $\mathrm{B2}$

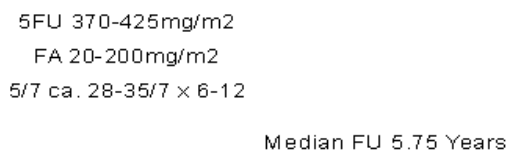

Figure 5. The IMPACT Trial for Stage 11 Colon Cancer

However IMPACT trial did not experience that patients receiving 5-FU/LV a significant increase in DFS or OS

Hence the American Society of Clinical Oncology (ASCO) does not recommend the routine use of adjuvant chemotherapy in stage II colon cancer, unless there are specific risk factors: poorly-differentiated histology, T4 lesions, bowel perforation and inadequately sampled lymph nodes.

\section{Place of novel agents}

A study done to assess the benefit of adding biological agents in adjuant setting was done in AVANT a randomised phase III study to assess effect of Bevacizumab(BEV) either with XELOX or FOLFOX (15). This data showed that the more patients randomised to either of the two arms including BEV had recurrent disease than those who received FOLFOX4 alone, although only the comparison between FOLFOX4-bevacizumab and FOLFOX4

Figure 6. The AVANT Trial - Addition of Bevacizumab for Colon Cancer

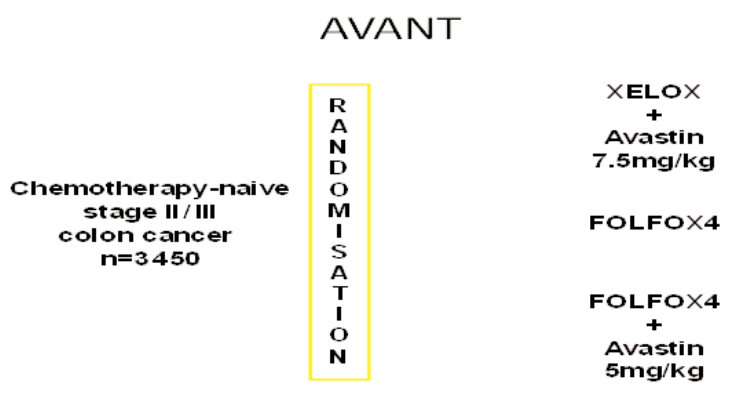

\section{Adjuvant therapies for large bowel cancer}

approached statistical significance. The primary endpoint of the AVANT study was not met (fig-6). BEV does not prolong DFS when added to either FOLFOX4 or XELOX in patients with stage III colon cancer.

It was concluded that there is no evidence of clinical benefit following addition of EGF or VEGF inhibitors to chemotherapy in the adjuvant setting

\section{When should adjuvant treatment be started?}

The best time to start adjuvant chemotherapy is within 6-8 weeks after surgery. A Swedish study in patients with stage III cancer, reported worse survival for patients who started adjuvant treatment more than eight weeks after surgery, when compared with those who started treatment after less than eight weeks. Moreover, the SAFFA trial reported significantly inferior survival for those patients who delayed starting adjuvant chemotherapy more than eight weeks [16].

\section{New directions for adjuvant therapy}

Molecular markers, such as microsatellite instability and defects in the DNA mismatch repair system, intratumoral levels of various enzymes involved in fluorouracil activation and metabolism, such as thymidylate synthase, dihydropyrimidine dehydrogenase and thymidine phosphorylase, are used to stratify prognostic groups. It will use predictive assays to select chemo responsive patients.

\section{Molecular stratification of prognostic}

\section{Conclusion}

- 5FU improves DFS and OS in stage III

- 5FU gives marginal improvement in stage II (OS)

- Infused 5FU is as effective as bolus 5FU

- Capecitabine is as effective as 5FU

- 3 months adjuvant chemotherapy may be equivalent to 6 months

- Irinotecan does not improve survival

- Oxaliplatin improves DFS

- No evidence of clinical benefit following addition of EGF or VEGF inhibitors to chemotherapy.

\section{References}

1. Buyse M, Zeleniuch-jacquotte A, Chalmers TC. Adjuvant therapy of colorectal cancer. Why we still don't know. JAMA. 1988; 259: 3571-8.

2. Moertel CG, Fleming TR, Macdonald JS, et al. Fluorouracil plus levamisole as effective adjuvant therapy after resection of stage III colon carcinoma: a final report. Ann Intern Med. 1995; 122: 321-6.

3. Efficacy of adjuvant fluorouracil and folinic acid in 
colon cancer. International Multicentre Pooled Analysis of Colon Cancer Trials (IMPACT) investigators. Lancet. 1995; 345: 939-44.

4. Haller DG, Catalano PJ, Macdonald JS, et al. Phase III study of fluorouracil, leucovorin, and levamisole in high-risk stage II and III colon cancer: final report of Intergroup 0089. J Clin Oncol. 2005; 23: 8671-8.

5. Andre T, Colin P, Louvet C, et al. Semimonthly versus monthly regimen of fluorouracil and leucovorin administered for 24 or 36 weeks as adjuvant therapy in stage II and III colon cancer: results of a randomized trial. J Clin Oncol. 2003; 21: 2896-903.

6. Saini A, Norman AR, Cunningham D, et al. Twelve weeks of protracted venous infusion of fluorouracil (5-FU) is as effective as 6 months of bolus 5-FU and folinic acid as adjuvant treatment in colorectal cancer. Br J Cancer. 2003; 88: 1859-65.

7. Twelves C, Wong A, Nowacki MP, et al. Capecitabine as adjuvant treatment for stage III colon cancer. N Engl J Med. 2005; 352: 2696-704.

8. André T, Boni C, Mounedji-boudiaf L, et al. Oxaliplatin, fluorouracil, and leucovorin as adjuvant treatment for colon cancer. N Engl J Med. 2004; 350: 2343-51.

9. Wolmark N, Wieand S, Kuebler JP, et al. A phase III trial comparing FULV to FULV + oxaliplatin in stage II or III carcinoma of the colon: Results of NSABP Protocol C-07. J Clin Oncol. 2005; 23(suppl): Abstract 3500.

10. Saltz LB, Niedzwiecki D, Hollis D, et al. Irinotecan plus fluorouracil/leucovorin (IFL) versus fluo- rouracil/leucovorin alone (FL) in stage III colon cancer (intergroup trial CALGB C89803). J Clin Oncol 2004; 22(suppl): Abstract 3500.

11. Van Cutsem E, Labianca R, Hossfeld G, et al. Randomized phase III trial comparing infused irinotecan/5-fluorouracil (5-FU)/folinic acid (IF) versus 5-FU/FA (F) in stage III colon cancer patients (pts). Proc Am Soc Clin Oncol 2005; 23: 1090s.

12. Schmoll HJ, Cartwright T, Tabernero J, et al. Phase III trial of capecitabine plus oxaliplatin as adjuvant therapy for stage III colon cancer: a planned safety analysis in 1,864 patients. J Clin Oncol. 2007; 25: 102-9.

13. Gray RG, Barnwell J, Hills R, et al. QUASAR: a randomized study of adjuvant chemotherapy vs observation including 3238 colorectal cancer patients. Proc Am Soc Clin Oncol. 2004; 22: Abstract 3501.

14. Efficacy of adjuvant fluorouracil and folinic acid in B2 colon cancer. International Multicentre Pooled Analysis of B2 Colon Cancer Trials (IMPACT B2) Investigators. J Clin Oncol. 1999; 17: 1356-63.

15. De gramont A, Van cutsem E, Schmoll HJ, et al. Bevacizumab plus oxaliplatin-based chemotherapy as adjuvant treatment for colon cancer (AVANT): a phase 3 randomised controlled trial. Lancet Oncol. 2012; 13: 1225-33.

16. Chau I, Norman AR, Cunningham D, et al. A randomised comparison between 6 months of bolus fluorouracil/leucovorin and 12 weeks of protracted venous infusion fluorouracil as adjuvant treatment in colorectal cancer. Ann Oncol. 2005; 16: 549-57. 